Received: 21 March 2017

Accepted: 14 August 2017

Published online: 07 September 2017

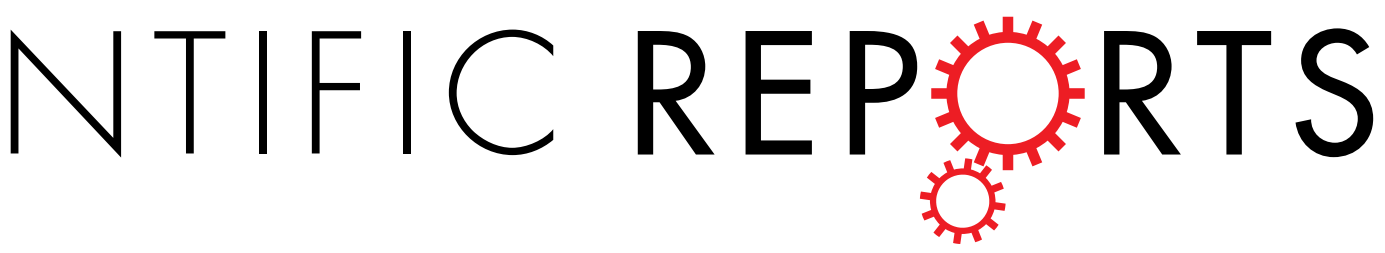

\title{
Quantitative imaging of anti-phase domains by polarity sensitive orientation mapping using electron backscatter diffraction
}

\author{
G. Naresh-Kumar ${ }^{1}$, A. Vilalta-Clemente ${ }^{2}$, H. Jussila ${ }^{3}$, A. Winkelmann ${ }^{4}$, G. Nolze ${ }^{5}$, S. Vespucci ${ }^{1}$, \\ S. Nagarajan ${ }^{3}$, A. J. Wilkinson $\mathbb{1}^{2}$ \& C. Trager-Cowan ${ }^{1}$
}

Advanced structural characterisation techniques which are rapid to use, non-destructive and structurally definitive on the nanoscale are in demand, especially for a detailed understanding of extended-defects and their influence on the properties of materials. We have applied the electron backscatter diffraction (EBSD) technique in a scanning electron microscope to non-destructively characterise and quantify antiphase domains (APDs) in GaP thin films grown on different (001) Si substrates with different offcuts. We were able to image and quantify APDs by relating the asymmetrical intensity distributions observed in the EBSD patterns acquired experimentally and comparing the same with the dynamical electron diffraction simulations. Additionally mean angular error maps were also plotted using automated cross-correlation based approaches to image APDs. Samples grown on substrates with a $4^{\circ}$ offcut from the [110] do not show any APDs, whereas samples grown on the exactly oriented substrates contain APDs. The procedures described in our work can be adopted for characterising a wide range of other material systems possessing non-centrosymmetric point groups.

Various material properties such as piezoelectricity, spontaneous polarisation, and plasticity are directly dependent on the crystal structure, and any form of deviation from their perfect crystal lattice could significantly alter their fundamental behaviour ${ }^{1}$. Producing defect free materials is a challenging task especially in the case of heteroepitaxial thin film growth. Irrespective of the substrates, the growth plane, or the growth conditions employed, extended defects such as dislocations, stacking faults and grain boundaries are generally observed in the as-grown layers. In addition to these commonly observed defects; inversion domains (IDs), antiphase domains (APDs) and antiphase boundaries (APBs) have also been identified in several materials; examples include, layered perovskite structured materials ${ }^{2,3}$ compound semiconductors $^{4-6}$, metallic superlattices ${ }^{7}$ and shape memory alloys ${ }^{8}$. Integrating the functionalities of all of the previously listed materials on a silicon platform is highly sought after to satisfy the demanding requirements for the next few generations of electronic and optoelectronic devices ${ }^{9}$. For example, monolithic integration of $\mathrm{A}^{\mathrm{III}}-\mathrm{B}^{\mathrm{V}}$ compounds on $\mathrm{Si}$ substrates would provide high-efficient, low-cost multi junction solar cells compatible with CMOS technology. Optimising the performance of such devices will require pioneering growth, and fabrication supported by characterisation techniques for a detailed understanding of defects. Often extended defects are electrically active ${ }^{10}$ and are problematic for minority carrier devices, such as $\mathrm{GaAs}$ solar cells, AlGaN-based ultra violet light emitting diodes, transistors and $\mathrm{SiC}$ power devices as well as $\mathrm{LaSrMnO}_{3}$ based spintronic devices ${ }^{3}$. This is why structural characterisation techniques which are simultaneously rapid to use, non-destructive and structurally definitive on the nanoscale become a prerequisite.

In this article, we demonstrate a novel application of electron backscatter diffraction (EBSD $)^{11}$ in a field emission scanning electron microscope (FE-SEM) to image and quantify APDs in a single crystalline GaP thin film grown on Si substrates. We have chosen $\mathrm{GaP}$ as an example to validate the applicability of using EBSD to

${ }^{1}$ Department of Physics, SUPA, University of Strathclyde, Glasgow, G4 ONG, UK. ${ }^{2}$ Department of Materials, University of Oxford, Parks Road, Oxford, OX1 3PH, UK. ${ }^{3}$ Department of Electronics and Nanoengineering, Aalto University, FI-00076, Aalto, Finland. ${ }^{4}$ Bruker Nano GmbH, Am Studio 2D, 12489, Berlin, Germany. ${ }^{5}$ BAM, Federal Institute for Materials Research and Testing, Unter den Eichen 87, 12205, Berlin, Germany. Correspondence and requests for materials should be addressed to G.N.-K. (email: naresh.gunasekar@strath.ac.uk) 
(a)

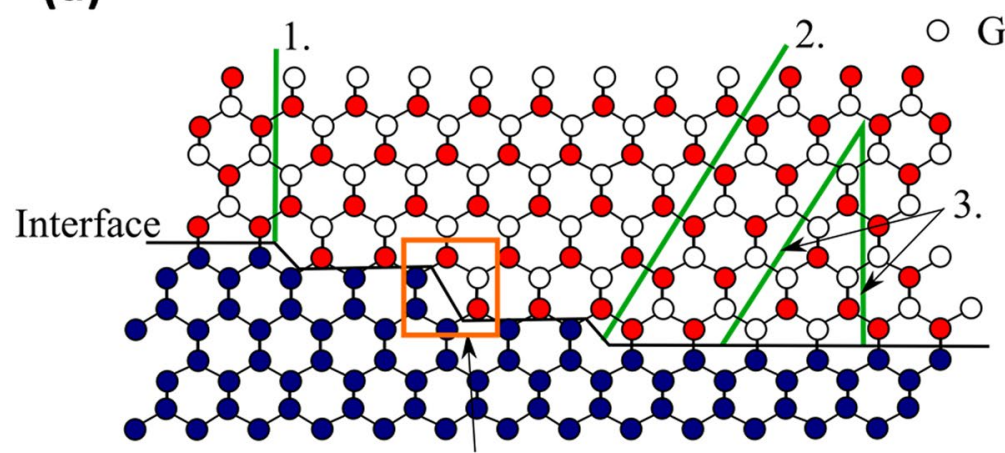

- Silicon atom

- Phosphorus atom

O Gallium atom

4.
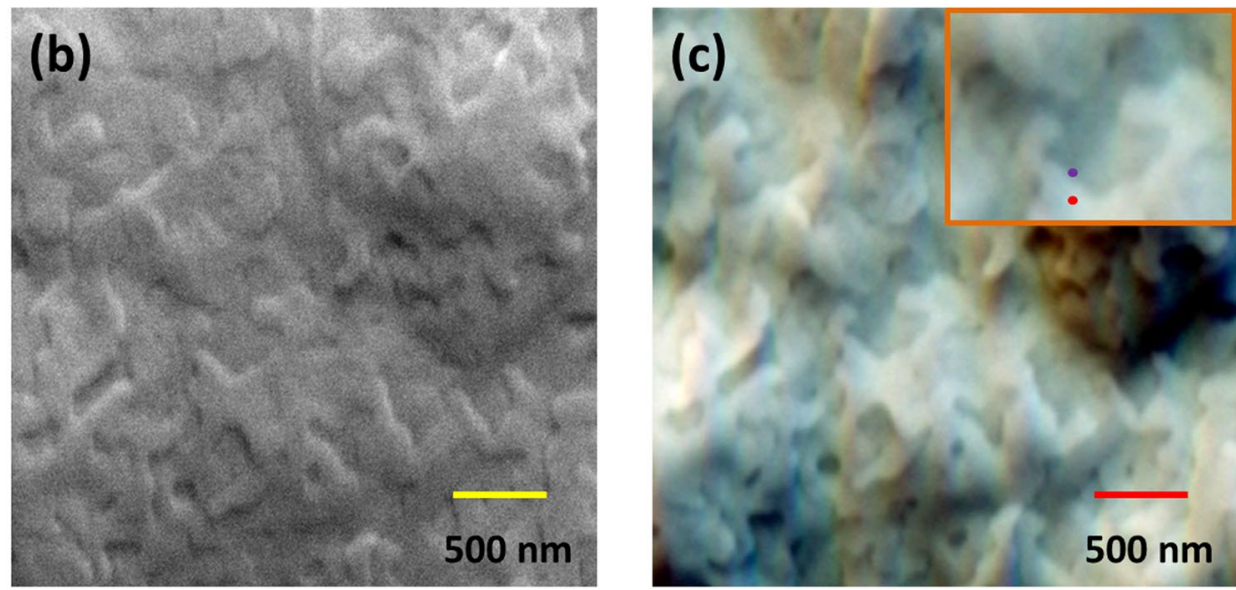

Figure 1. Antiphase domains in $\mathrm{GaP}$ on Si substrates. (a) Ball and stick model illustrating the formation and annihilation of antiphase boundaries (APBs) in GaP grown on a (001) $\mathrm{Si}$, (1.) APBs parallel to (110) due to sub-lattice occupation disorder, (2.) APBs along the $\{111\}_{\mathrm{Si}}$ due to monoatomic steps, (3.) annihilation of APBs along (111) and (110) and (4.) annihilation of APBs due to diatomic steps. (b) Plan-view secondary electron image and (c). Plan-view backscattered electron image both acquired at a sample tilt of $70^{\circ}$ from the same region of the surface of a $70 \mathrm{~nm} \mathrm{GaP} \mathrm{film} \mathrm{grown} \mathrm{on} \mathrm{an} \mathrm{(001)} \mathrm{Si} \mathrm{substrate} \mathrm{(miscut}<0.1^{\circ}$ ). The inset shows areas marked with a red dot and a purple dot which may well be regions with two orientations corresponding to a difference in the location of cation atoms (eg. Ga) and anion atoms (P) in the two sub-lattices as expected in the case of APDs.

characterise APDs; nonetheless our non-destructive, and nanoscale technique can be adopted for other material systems, especially those having non-centrosymmetric point groups $\mathrm{s}^{2-8}$. Please note optimal experimental conditions have to be chosen while using EBSD for samples with low thermal conductivity ${ }^{12}$.

\section{Antiphase domains in GaP}

When a non-centrosymmetric polar material such as zincblende $\mathrm{GaP}(\overline{4} 3 \mathrm{~m})$ is epitaxially grown on a centrosymmetric non-polar material $\mathrm{Si}(m \overline{3} \mathrm{~m})$, two equivalent orientations corresponding to a difference in the location of cation atoms (for e.g., Ga) and anion atoms (P) in the two sub-lattices can be formed leading to the creation of APDs. The boundary separating the domains of different sub-lattice location is defined as the APB. Formation of APDs in GaP epilayers grown on Si surfaces is mainly affected by the surface steps of the Si substrate, see Fig. 1a. Atomically clean, vicinal (001) Si surfaces are generally dominated by monoatomic steps due to their low formation energy ${ }^{13}$. The monoatomic steps have a width of $1 / 4$ of the Si lattice constant due to the diamond cubic structure (space group $\mathrm{Fd} \overline{3} \mathrm{~m}$ ) with two tetrahedrally connected $\mathrm{Si}$ atoms in each primitive cell separated by $1 / 4$ of the width of the unit cell in all three dimensions. Typically, these monoatomic steps along the growth direction [001] are assumed to be responsible for the formation of $\mathrm{APBs}$ at the $\mathrm{Si} / \mathrm{GaP}$ interface, leading to their propagation along the (011) plane (see Fig. 1a-1) or the (111) plane (see Fig. 1a-2) ${ }^{14}$. APBs can also be formed due to the sub-lattice occupation disorder at the Si/GaP interface parallel to $\{110\}_{\mathrm{Si}}$ or $\{111\}_{\mathrm{Si}}$, where $\mathrm{Ga}$ and $\mathrm{P}$ atoms in the region to the left of the boundary sit in different sub-lattices from the region to the right (see Fig. 1a-3). In simple geometric terms, the $\mathrm{GaP}$ crystal appears to be rotated by $90^{\circ}$ around[001] between the sides of the APBs. APDs could be eliminated either by self-annihilation along the $\{111\}_{\mathrm{Si}}$, by obtaining a uniform monolayer of either Ga or P nucleation on Si by using optimised growth conditions, or by growing on Si surfaces with diatomic steps (see Fig. 1a-4 $)^{15-18}$. Nonetheless, it is quite a challenging task to produce smooth GaP surfaces especially below the 


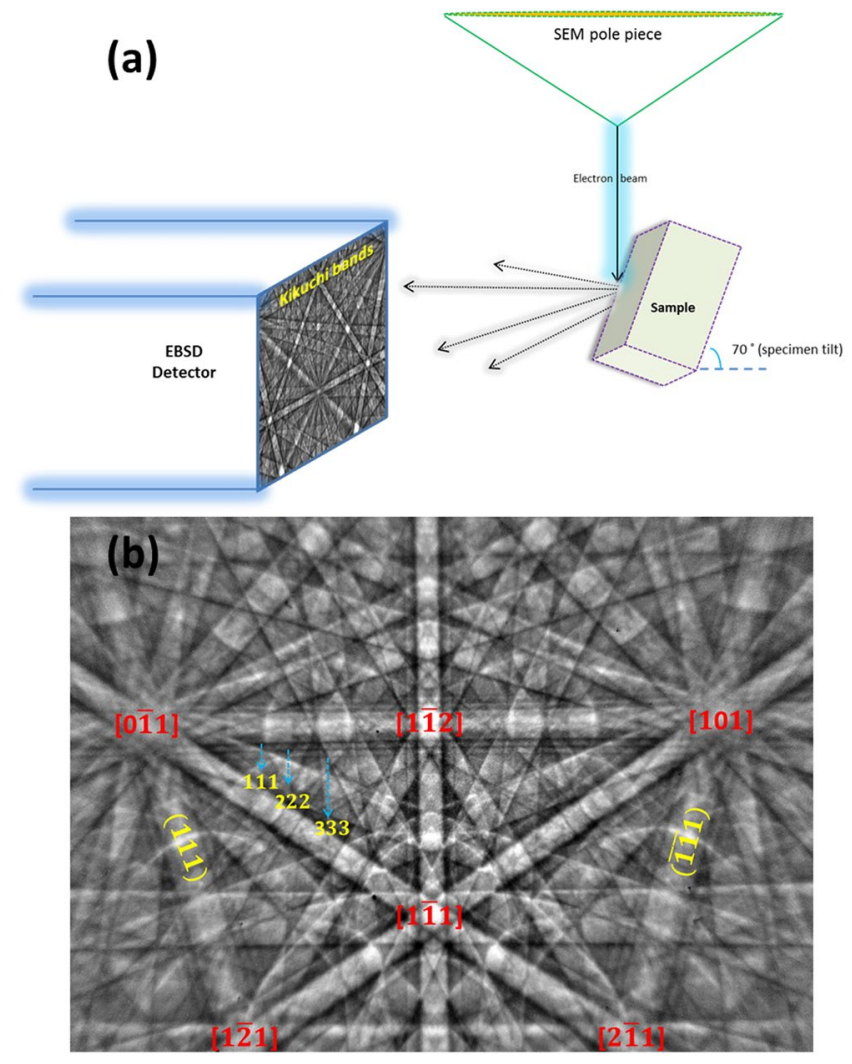

Figure 2. EBSD experimental setup. (a) Schematic of a standard EBSD detection geometry and (b) EBSD pattern from a GaP thin film marked with some major lattice planes and well-visible zone axes.

critical thickness (of order $90 \mathrm{~nm}$ for $\mathrm{GaP}$ grown on $(001) \mathrm{Si}^{19}$ ), the sample surface can exhibit "twisted-line" like features. The twisted-line morphology can result from the surface step structures from the Si substrate and is associated with $\mathrm{APBs}^{18}$. The sample morphology exhibiting the twisted-lines are shown in the secondary electron image (SE) as well as in the backscattered electron image (BSE) acquired in a forward scattered geometry, also referred to as a forescattered electron (FSE) image, see Fig. $1 \mathrm{~b}$ and $\mathrm{c}$ respectively. The images were acquired from a $70 \mathrm{~nm}$ thick GaP film grown on an $(001) \mathrm{Si}$ substrate (miscut $<0.1^{\circ}$ ) by metalorganic vapor phase epitaxy. The inset in Fig. 1c is marked with a red dot and a purple dot which may well be regions with two orientations corresponding to a difference in the location of cation atoms (eg. Ga) and anion atoms $(\mathrm{P})$ in the two sub-lattices as expected in the case of APDs.

Although stress induced extended defects such as dislocations are unlikely to form below the critical thickness, non-optimised growth conditions can generate stacking faults. The formation of stacking faults may be due to the coalescence of 3-D islands produced as a result of a lack of charge neutrality along the Si-GaP interface. Possessing equal numbers of $\mathrm{Si}-\mathrm{Ga}$ bonds and $\mathrm{Si}-\mathrm{P}$ bonds is essential to maintaining charge neutrality which is needed to initiate $2-\mathrm{D}$ growth, which in turn is a requirement for producing smooth surfaces ${ }^{17-20}$. In the present work we will limit the discussion to imaging APDs in our samples. Nonetheless, we note that the set-up used in this work (i.e. electron diffraction imaging with high resolution SEM) is also suited for observing stacking faults formed due to the coalescence of 3-D islands.

Several methods have previously been reported for detecting APDs in GaP, examples include anisotropic etching, X-ray diffraction (XRD) ${ }^{21}$, reflectance anisotropy spectroscopy (RAS) ${ }^{22,23}$ and transmission electron microscopy (TEM) $)^{4,14,24,25}$. All these methods are either indirect or destructive and time consuming or cannot provide statistically significant information on APDs. In contrast to all the previously reported techniques, the scanning electron microscope (SEM) based electron diffraction techniques of EBSD and electron channelling contrast imaging (ECCI) provide the capability of rapid and non-destructive characterisation, giving accurate wide area crystallographic information with resolution down to the order of tens of nanometres ${ }^{11,26-29}$.

EBSD in a scanning electron microscope. In EBSD, an electron beam is incident on a sample which is typically tilted at an angle of $70^{\circ}$ towards the detector. Classically the detector is a phosphor screen which captures backscattered electrons from the sample. The quasi-elastically backscattered part produce a diffraction signal, generally referred to as a backscattered Kikuchi diffraction (BKD) pattern or electron backscatter diffraction (EBSD) pattern. The EBSD pattern also holds all the inelastically scattered electrons (background signal). The schematic of the detection geometry and the experimental setup is shown in Fig. 2a. As a first approximation, the visible bands in an EBSD pattern can be interpreted by the angular distribution of the Bragg-reflected electrons coming from the lattice planes $(h k l)$ of a crystalline sample. Due to the cylindrical symmetry of the Bragg 
reflection conditions with respect to the lattice plane normal, diffraction cones are formed. When the diffraction cones (Kossel cones) intersect the phosphor screen, nearly straight lines (Kikuchi lines) are seen due to the shallow angle of the Bragg-diffracted cones. Thus the EBSD pattern can be used to directly measure the crystal's orientation. An example EBSD pattern acquired from a GaP thin film is shown in Fig. 2b. It is these diffraction patterns which hold the structural information of the crystalline specimen. By moving a focused electron beam point by point across a grid of positions on the sample surface, phase distribution or orientation maps can be derived providing a quantitative representation of the local microstructure.

EBSD patterns represent the gnomonic projection of the diffraction signal. Any rotation of a crystal will produce a corresponding rotation in the EBSD pattern. The simplified geometrical model described does not consider the intensity associated with the EBSD patterns which also bear the information about the crystal structure. In particular, the kinematical theory of electron diffraction ${ }^{11}$ does not account for multiple scattering of electrons inside the crystal. Therefore for a quantitative calculation of the EBSD patterns, one has to consider the strong effects of multiple scattering and absorption, and hence dynamical theory of electron diffraction ${ }^{11,30}$ becomes mandatory. Detailed reviews on various models for EBSD pattern simulations, limitations of kinematical theory and the physics behind dynamical theory calculations can be found elsewhere ${ }^{11,30-32}$.

For the sake of simplicity, all the commercial EBSD systems determine crystal orientations with respect to the centrosymmetric Laue groups of a phase, although EBSD patterns are sensitive to the point-group symmetry of a crystal. Space group determination is also possible in certain cases which can be time consuming ${ }^{33}$. Advancement in computing power has made it possible to simulate the dynamical electron diffraction signal and compare it with the experimental patterns for the analysis of crystal orientation and their relationship with phase transformations and chirality determination ${ }^{34-36}$. In our present work, we have used the ESPRIT DynamicS (Bruker Nano) software which implements the Bloch wave approach for calculating the simulated EBSD patterns $s^{30}$. Typically 1000 or more reciprocal lattice vectors $h k l$ (diffraction orders) are taken into account and they are selected according to the reciprocal lattice vector length $\mathrm{d}^{*}{ }_{\mathrm{hkl}}=1 / \mathrm{d}_{\mathrm{hkl}}$ (typically $\mathrm{d}_{\mathrm{hkl}}{ }_{\mathrm{k} l}<1 / 0.05 \ldots 1 / 0.035 \mathrm{~nm}^{-1}$ ) and the relative strength with respect to the largest structure factor amplitude $|\mathrm{F}|_{\text {max }}$ (typically $<10 \%$ ). For interpreting the EBSD patterns and for plotting the orientation maps, the crystal orientations were parameterised using the ZXZ-type Euler angles $\left(\varphi_{1}, \phi, \varphi_{2}\right)$ in the Bunge convention ${ }^{37}$. For quantifying the agreement between two EBSD patterns, we have used the normalised cross-correlation coefficient, $\mathrm{r}^{38,39}$.

EBSD patterns exhibit different distributions of intensity; an asymmetry in the intensity profile across a Kikuchi bands can be due to either the excess-deficiency effect ${ }^{39}$ or due to the breakdown of Friedel's rule for the intensity at the symmetrically located Bragg angle locations, leading to $I_{h k l} \neq I_{\bar{h} \bar{l} l}{ }^{32}$. It is this intensity asymmetry, due to the point-group sensitivity of EBSD, which we are going to exploit to image APDs. In contrast, the excess-deficiency effect is a result of the geometry of the measurement which affects the differential cross section for inelastic scattering ${ }^{40}$. The influence of the excess-deficiency asymmetry depends on the relative orientation of the Kikuchi bands with respect to the incident beam direction and can be minimized by careful selection of the sample orientation ${ }^{41}$. Please note the reliable discrimination of the Kikuchi bands intensity asymmetry is only possible when the intensity shift due to the breakdown of Friedel's rule is considerably larger than the excess-deficiency effect.

\section{Results and Discussion}

In non-centrosymmetric zincblende structures such as GaP, there is an asymmetric stacking sequence of Ga atoms and $\mathrm{P}$ atoms along $\langle 111\rangle$ and $\langle\overline{1} \overline{1} \overline{1}\rangle$ (see Fig. 1a) ${ }^{33}$, ${ }^{39}$. Hence, Kikuchi bands formed from non-centrosymmetric lattice planes like $\{111\}$ and $\{\overline{1} \overline{1} \overline{1}\}$ show an asymmetry in the intensity profile (i.e. the intensity maximum is marginally shifted out of the centre of the Kikuchi band) which allows the observation of the inversion symmetry ${ }^{42}$, 43 . The effect of an asymmetry on all the polar lattice planes is demonstrated in Fig. 3. Figure $3 \mathrm{a}$ and $\mathrm{b}$ show the experimental EBSD pattern recorded from areas marked with a red dot and a purple dot (see inset of Fig. 1c) respectively. The corresponding simulated patterns are shown in Fig. 3d and e. We have used the automated best fit EBSD pattern matching approach ${ }^{39}$ based on the normalised cross-correlation coefficient $\mathrm{r}^{38}$ to compare the experimental EBSD patterns and the simulated EBSD patterns. On a casual assessment, both the experimental as well as the simulated EBSD patterns looks very similar. However, on careful inspection one can see the differences in the intensity associated with the Kikuchi bands, especially along the $\{111\}$ bands where the higher intensity is towards either the top or bottom of the respective Kikuchi band edge which indicates the $\{111\}$ and $\{\overline{1} \overline{1} \overline{1}\}$. This can be seen clearly in the normalised intensity difference $\left(\mathrm{I}_{\mathrm{a}}-\mathrm{I}_{\mathrm{b}}\right) /\left(\mathrm{I}_{\mathrm{a}}+\mathrm{I}_{\mathrm{b}}\right)$ (Fig. 3c) between the two experimental EBSD patterns (Fig. $3 \mathrm{a}$ and $\mathrm{b}$ ). The correct orientation with respect to the point-group symmetry $(\overline{4} 3 \mathrm{~m})$ is determined by best-fitting the experimental and the respective simulated patterns which provide the higher cross-correlation coefficient $r$. The Euler angle $\varphi_{2}$ provides the last rotation around Z-axis which is equivalent to a rotation around [001] or the $c$-axis (which is also the epitaxial growth direction of our sample). The experimental EBSD pattern shown in Fig. $3 \mathrm{a}$ is compared with the simulated pattern shown in Fig. $3 \mathrm{~d}$ and results in $r$ of 0.627 and the Euler angle $\varphi_{2}$ of $180.9^{\circ}$. But, when the Euler angle $\varphi_{2}$ for the simulated EBSD pattern is changed to $270.9^{\circ}$ (i.e. $180.9^{\circ}+90^{\circ}$ ) the value of $r$ is decreased to 0.599 , representing a small but significant discrepancy from the experimental EBSD patterns. The same approach is repeated for the experimental pattern shown in Fig. $3 \mathrm{~b}$ and the respective simulated EBSD pattern shown in Fig. 3e. In this case, when the Euler angle $\varphi_{2}$ is of $180.9^{\circ}$ the value of $\mathrm{r}$ is 0.601 (decreased when compared to Fig. 3a and d), but when the Euler angle $\varphi_{2}$ is of $270.9^{\circ}$ the value of $r$ is increased to 0.627 (increased when compared to Fig. $3 \mathrm{a}$ and d). Therefore, for the experimental pattern shown in Fig. 3a, the correct experimental Euler angle $\varphi_{2}$ is $180.9^{\circ}$ whereas for the pattern showed in Fig. 3b, the correct experimental Euler angle $\varphi_{2}$ is $270.9^{\circ}$.

The dissimilarity between the Kikuchi patterns with two different Euler angles can be best seen in Fig. 3c,f which show the normalised intensity difference (by using the same scaling) between the two experimental 

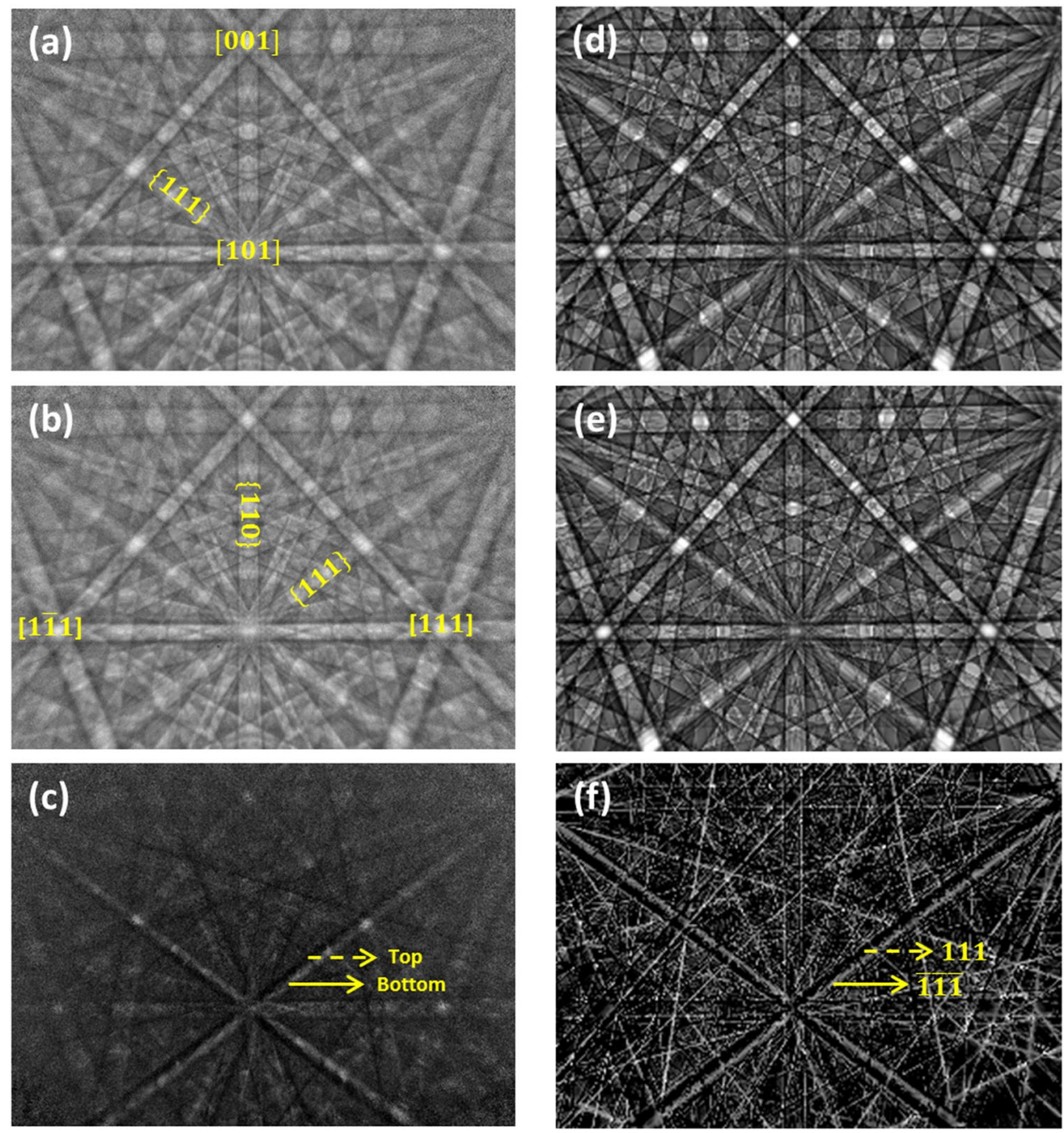

Figure 3. Comparison of experimental and simulated EBSD patterns. (a) Experimental EBSD pattern from the red dotted area, see Fig. 1c (for e.g.; the location of cation (Ga) atoms), with $r=0.627$ and $\varphi_{2}=180.9^{\circ}$ and (b) from the purple dotted area (for e.g.: $\mathrm{P}$ site with $\mathrm{r}=0.627$ and $\left.\varphi_{2}=270.9^{\circ}\right)$ and $(\mathbf{d}$ and $\mathbf{e})$ the corresponding dynamical simulations. (c) Normalised difference intensity image $\left(\mathrm{I}_{\mathrm{a}}-\mathrm{I}_{\mathrm{b}}\right) /\left(\mathrm{I}_{\mathrm{a}}+\mathrm{I}_{\mathrm{b}}\right)$ of the two experimental patterns and (f) normalised difference intensity image of the two simulated patterns. The strong asymmetric intensity difference between the $\{111\}$ and $\{\overline{1} \overline{1} \overline{1}\}$ in the normalised difference intensity images clearly indicates the crystal structure rotation by $90^{\circ}$ confirming the presence of APDs in the GaP thin film.

(Fig. 3a,b) and the corresponding simulated patterns (Fig. 3d,e). The sufficient intensity difference between polar planes such as $\{111\}$ and $\{\overline{1} \overline{1} \overline{1}\}$ clearly indicates the $90^{\circ}$ sub-lattice rotation confirming the presence of APDs in the $\mathrm{GaP}$ thin film. As all crystal directions normal to an even fold rotation axis are non-polar ${ }^{41}$, all the Kikuchi bands of $\{\mathrm{hk} 0\}$, for e.g. $\{110\}$ disappear in the normalised difference intensity image (see Fig. 3c,f). In our case the asymmetric intensities are not due to excess-deficiency effects, nonetheless care has to be taken while acquiring the EBSD patterns as the strength of the excess-deficiency effects depends on the relative orientation of the Kikuchi bands with respect to the incident beam direction. Bands running normal to the incident beam direction (horizontal bands) show more of the excess-deficiency effects. Hence the Kikuchi bands of $\{111\}$ and $\{\overline{1} \overline{1} \overline{1}\}$ are acquired diagonally in Fig. 3 when compared to the indexed EBSD pattern shown in Fig. 2b.

Imaging BSE with an EBSD detector. Recently it was shown that it is possible to use the EBSD detector as an imaging device ${ }^{44-46}$ similar to a diode detector used in ECCI ${ }^{28,29,47}$. Basically, each pixel of the CCD camera operates as an individual backscattered electron detector and the intensity of electrons at a specific pixel is recorded at each point during a step by step scanning of the sample, helping to derive the microstructural information $^{46,48,49}$. We have utilised this feature and have defined regions which mainly covers the EBSD patterns formed by $\{111\}$ and $\{\overline{1} \overline{1} \overline{1}\}$ (e.g. see Fig. 3a,b) as our regions of interest (ROI). This enabled us to map the asymmetrical intensity variations thereby revealing the APDs by calculating the intensity asymmetry $\left(\mathrm{I}_{\mathrm{a}}-\mathrm{I}_{\mathrm{b}}\right) /\left(\mathrm{I}_{\mathrm{a}}+\mathrm{I}_{\mathrm{b}}\right)$ between two ROIs, corresponding to reflections related by inversion. Figure $4 \mathrm{a}$ shows the Kikuchi bands ROI asymmetry image derived from the post processing of the recorded EBSD patterns ${ }^{50}$. Bright and dark regions 

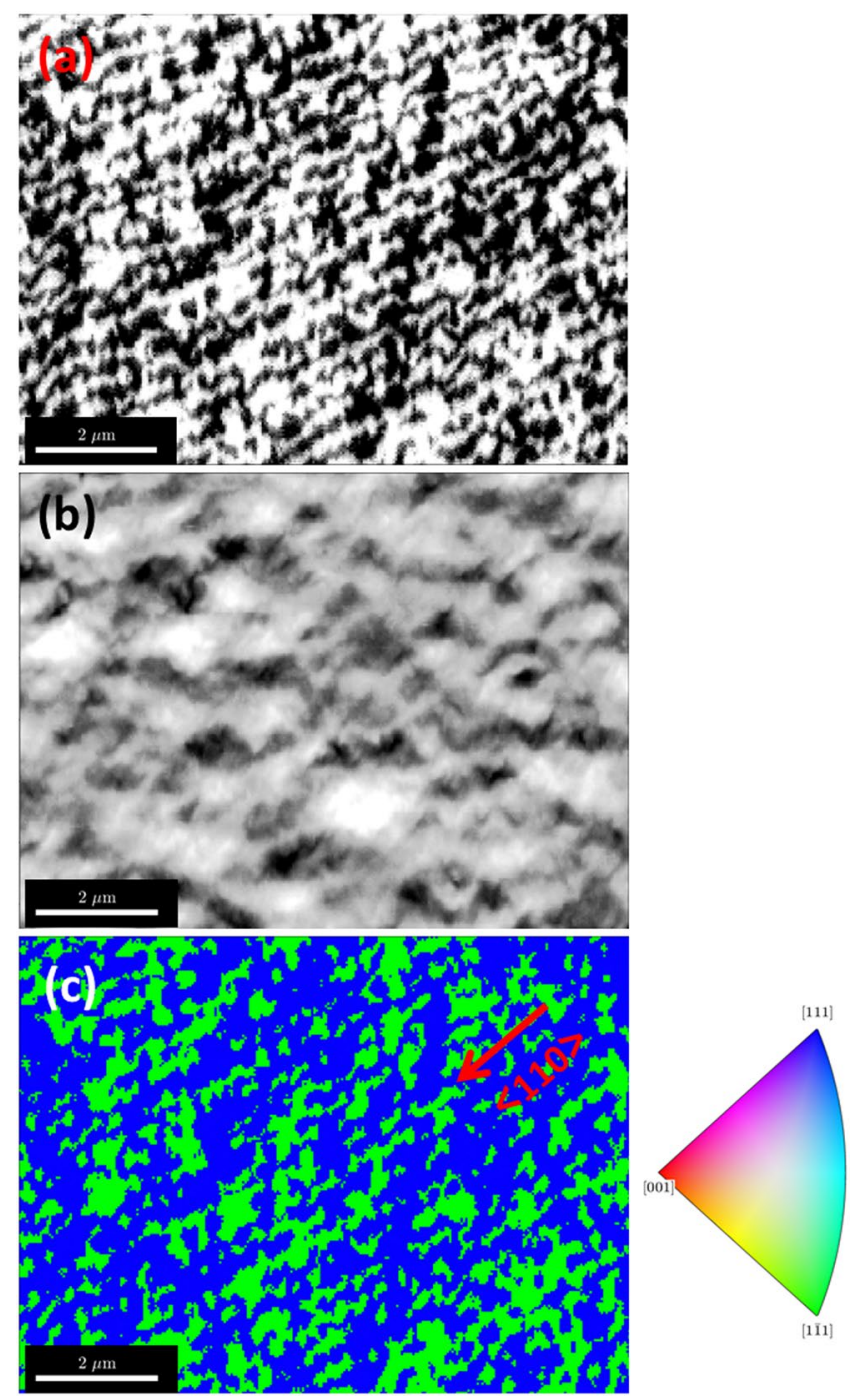

Figure 4. Imaging antiphase domains. (a) Region of interest asymmetry imaging from the $\{111\}$ bands produced from the background corrected EBSD patterns. The bright and dark regions indicate the two different pseudo-symmetric domains, (b) corresponding total intensity image of the raw EBSD patterns and (c) the EBSD inverse pole figure (IPF) map for the sample reference direction $[1,1,1]$ revealing the APDs. Regions with APDs are coloured green and blue according to the IPF colour key, which indicates the expected $90^{\circ}$ misorientation between the two possible domains. The step structures (of the order of $100 \mathrm{~nm}$ ) can also be seen along $\langle 110\rangle$.

correspond to opposite asymmetry values with an additional minor experimental offset caused by excess-deficiency effects and other intensity variations underlying the selected ROIs; and so allowing the respective APDs. The corresponding EBSD pattern quality image or in other words the total intensity image (Fig. 4b) shows the sample morphology, similar to the FSE image. Thus by choosing only a particular band as ROIs, quantitative microstructural information on the APDs can be obtained in a relatively simple way. This can be compared to the inverse pole figure (IPF) orientation maps as shown in Fig. 4c obtained from the same area using the automated pattern matching approach as discussed previously.

Inverse pole figure (IPF) orientation maps. The IPF specifies the crystallographic description of a specific sample direction, i.e. it displays which lattice direction $\langle u v w\rangle$ is parallel to the sample direction the IPF is assigned to. To reveal the APDs using different colours in the IPF colour key, we have chosen the unconventional sample direction $[1,1,1]$ as the reference (usually one selects $\mathrm{X}=[1,0,0], \mathrm{Y}=[0,1,0]$ or $\mathrm{Z}=[0,0,1]$ as the reference directions). The maps show that the more general reference direction $[1,1,1]$ is coloured by green regions, i.e. parallel to $\langle 1 \overline{1} 1\rangle$ or rotated by $90^{\circ}$ around [001] so that $\langle 111\rangle$ is parallel to the $[1,1,1]$ sample direction, the blue regions. The step pattern (arising due to the Si substrate steps) with a step width of $\approx 100 \mathrm{~nm}$, similar to the dimensions of the atomic steps on the $\mathrm{Si}$ surface, can also be clearly seen along the $\langle 110\rangle$. This confirms the formation of APDs due to the steps along the (110) as explained previously (see Fig. 1a). The percentage of APDs can be estimated by calculating the areas of the green regions from a scanned area of $\approx 75 \mu \mathrm{m}^{2}$, which accounts for $\approx 50 \%$. The density of the APBs is estimated to be $\approx 2.6 \mu \mathrm{m}^{-1}$. The shapes of the APDs seem to be non-uniform and 

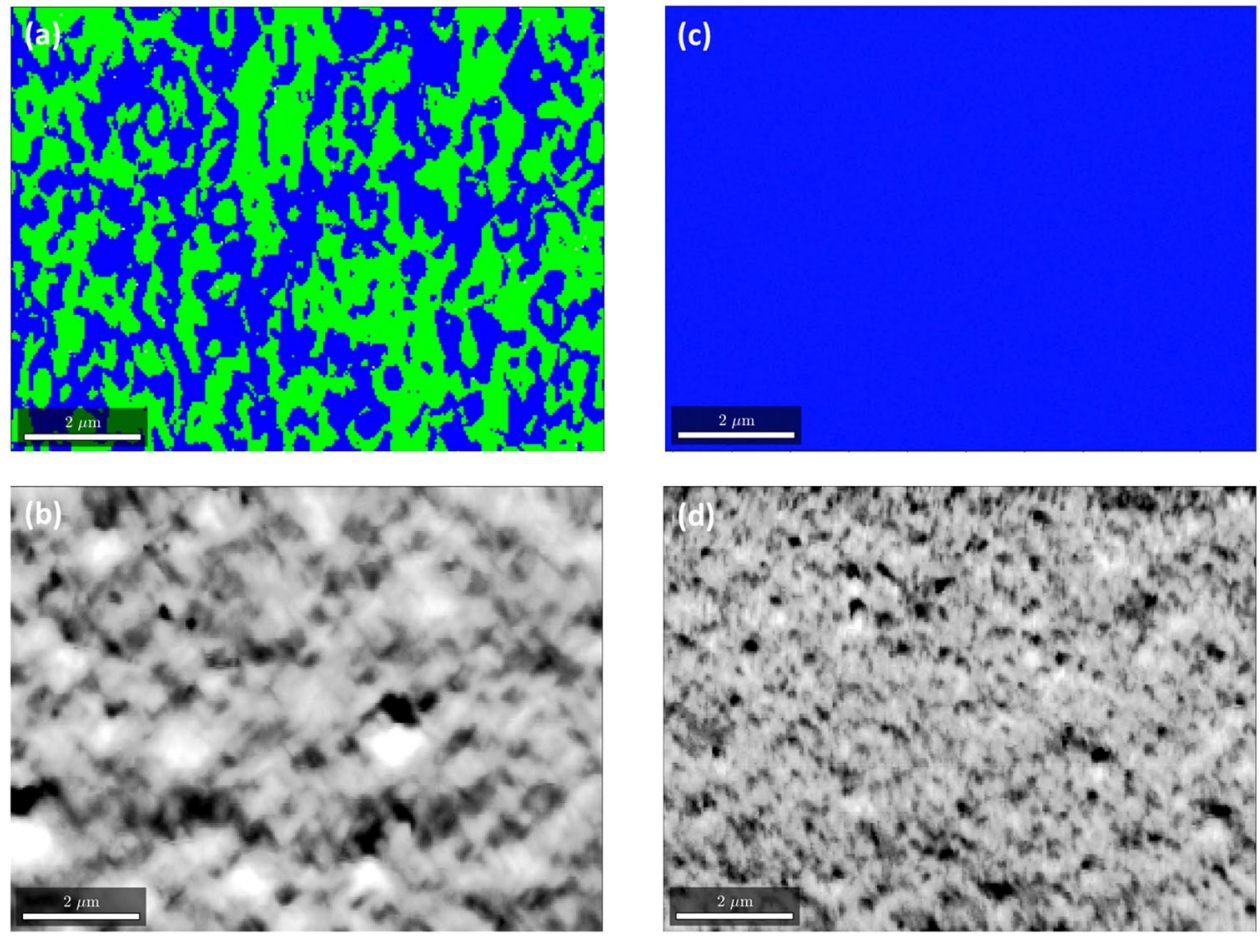

Figure 5. EBSD Inverse Pole Figure (IPF) maps of GaP thin films. (a) Grown on a different (001) Si substrate with similar growth conditions as the sample shown in Fig. 4.(b) corresponding BSE image derived from the EBSD patterns (c) IPF-map of GaP grown on a $4^{\circ}$ misoriented Si substrate, not showing any APDs and (d) its corresponding BSE image derived from the EBSD patterns. The colour keys for the IPF-maps in (a) and (c) are the same as shown in Fig. 4c.

appear to be narrow along $\langle 110\rangle$. Cross-section EBSD or 3-D EBSD could be useful for characterising the shape of the APDs for thicker samples. In order to check the reliability of our analysis, IPF maps were plotted for another $\mathrm{GaP}$ sample with similar growth conditions, however on a Si substrate from a different manufacturer (see Fig. 5a). The percentage of APDs is similar to the previous sample; with an APD content of $\approx 50 \%$ and an APB density of $\approx$ $2.7 \mu \mathrm{m}^{-1}$. Figure $5 \mathrm{~b}$, shows the corresponding EBSD pattern quality image revealing the sample morphology. We have also performed experiments on a $\mathrm{GaP}$ sample grown on a $4^{\circ}$ misoriented $\mathrm{Si}$ substrate which did not reveal any APDs (see Fig. 5c). This is expected because of the thermodynamically favored formation of bi-atomic steps on the Si substrates with a large $\left(>4^{\circ}\right)$ off-cut ${ }^{51,52}$ preventing the formation of APDs in the GaP layers. The sample morphology derived from the EBSD pattern quality map is displayed in Fig. $5 \mathrm{~d}$. The APB densities estimated from our present work are similar to numbers derived from Barrett et al. on GaAs epitaxially grown on (001) $\mathrm{Si}^{53}$.

Cross-correlation based high angular resolution EBSD. Since the $90^{\circ}$ lattice rotation around [001] makes it possible to image the APDs due to the asymmetric intensity in the Kikuchi bands, consequently it should also be possible to image them using cross-correlation based high angular resolution (HR)-EBSD ${ }^{54,55}$. In HR-EBSD all the experimental patterns within a map are compared to a user selected reference experimental EBSD pattern using cross-correlation of the pattern intensities and the position within a number of ROI (35 ROIs in our present case) with a band pass filter applied in the Fourier domain to remove high frequency noise and low frequency background intensity variations. Cross-correlation algorithms find the translation between two matched regions within the patterns being compared and extract a translation (shift) along both $\times_{1}$ and $\times_{2}$ directions using the cross-correlation peak position. In addition, the correlation peak height is used as a measure of how good the best pattern matching is ${ }^{56}$. Maps of the variation of elastic strain $\left(\varepsilon_{i j}\right)$ and lattice rotation $\left(\omega_{i j}\right)$ relative to that at the reference point (selected reference experimental EBSD pattern) can be generated using HR-EBSD with a very high precision of better than $10^{-4}$ rads (for rotation) and about $10^{-4}$ (for strain), respectively. More information about HR- EBSD cross-correlation analysis and its applications for strain and misorientations analysis are given in refs 54-57. In order to image the APDs, we have plotted the Mean Angular Error (MAE) map which is a quantitative measurement of unrealistic rotation measurements. Please note the $90^{\circ}$ rotation around [001] between the sides of the boundaries is not the actual crystal misorientations. The MAE maps, where the mean of the errors for each ROI, between the as measured shift of a particular ROI and the shift expected from the finally calculated rotation tensor for that same ROI, can be used to image APDs. Figure 6a shows the FSE image of a GaP film (same as shown in Fig. 4) taken prior to acquiring HR-EBSD maps and Fig. $6 \mathrm{~b}$ shows the corresponding IPF map plotted using the same procedure used to plot Fig. 4c. The cross correlation analysis of the HR Kikuchi patterns were conducted off-line and it is possible to choose the reference pattern from the area of interest of our choice. We have taken a reference pattern from the green region (APD region) marked with a red dot and plotted the MAE map; this can 

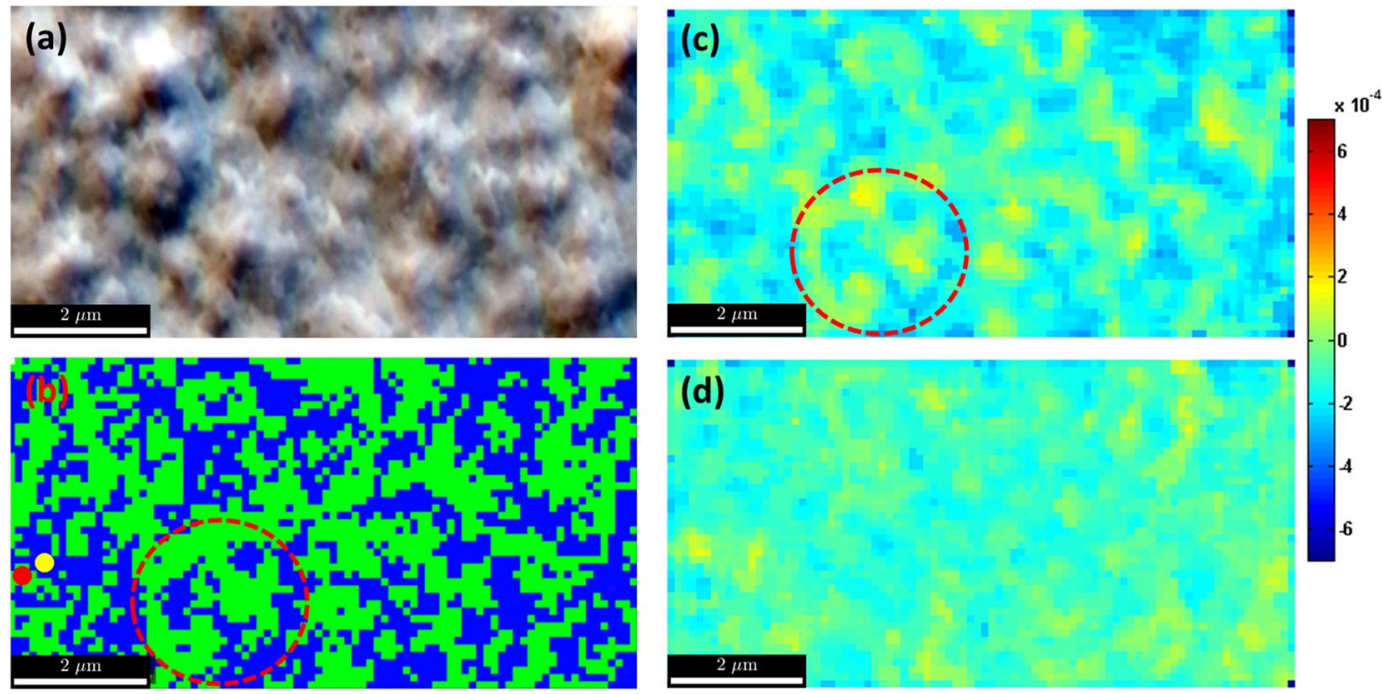

Figure 6. Comparison of IPF maps with cross correlation based MAE maps. (a) Forescatter image, (b) IPF map, (c) MAE (in radians) map plotted with green area in the IPF as a reference pattern, see red dot and (d)) MAE map with blue area as the reference pattern, see yellow dot.

be seen in Fig. 6c. A dotted red circle is marked in both Fig. $6 \mathrm{~b}$ and $\mathrm{c}$ to highlight the same area. We have also plotted the MAE map by choosing a reference pattern from the blue region (non-APD region) marked with a yellow dot. One can notice a broader distribution of MAE when the reference pattern is taken from the APD regions.

\section{Conclusion}

In summary, the asymmetrical intensity associated with the EBSD patterns acquired using backscattered electrons in a scanning electron microscope is used to image APDs in GaP thin films grown on Si substrates. We have used three approaches to quantify and image APDs, namely by (i) comparing the experimental pattern with the dynamically simulated pattern, (ii) orientation mapping (IPF maps) using the correct non-centrosymmetric point group by involving an automatic pattern matching approach and (iii) plotting mean angular error maps using cross-correlation based HR-EBSD. We have also tested our automatic pattern matching approach on GaP samples grown on different $S i$ substrates with and without APDs to show the reliability of our nanoscale, rapid and non-destructive approach on imaging APDs. The proposed analysis may well be generally applied for a wider range of other materials possessing non-centrosymmetric point groups.

\section{Methods}

Growth of GaP thin films. GaP films were grown on (001) Si substrates by metalorganic vapor phase epitaxy. The Si substrates were either exactly (001) oriented with a miscut $<0.1^{\circ}$ or intentionally miscut by $4^{\circ}$ towards $\langle 110\rangle$. Prior to the growth, the Si substrates were etched in $\mathrm{HF}: \mathrm{H}_{2} \mathrm{O}(1: 10)$ for 60 seconds to remove the native oxide followed by high temperature (i.e. $850^{\circ} \mathrm{C}$ ) in-situ annealing step in $\mathrm{H}_{2}$ environment. Tertiarybutylphosphine (TBP) and trimethylgallium (TMGa) were used as source materials. In order to ensure that the growth mode is as 2-D as possible, a two-step growth mode was used. First, a nominally $5 \mathrm{~nm}$-thick GaP nucleation layer (NL) was grown at $475^{\circ} \mathrm{C}$. Due to reduced ad atom surface mobility at lower growth temperatures, the surface coverage of the ad atoms is improved causing the NL to cover the Si surface. However, due to inefficient decomposition of the source materials at lower growth temperatures, the growth rate of NL is extremely slow, approximately $10 \%$ of that at $600^{\circ} \mathrm{C}$. Therefore, the growth temperature was ramped up to $700^{\circ} \mathrm{C}$ to grow a 70 -nm-thick GaP layer on top of the NL. Note that the thickness of the GaP layer was selected to be $70 \mathrm{~nm}$ because it is smaller than the critical thickness for misfit dislocation formation. The sample surface shows the RMS roughness value from the AFM analysis is $\approx 1.5 \mathrm{~nm}$ for a $2.5 \mu \mathrm{m}^{2}$ scanned area ${ }^{49}$.

FE- SEM beam conditions and EBSD experimental settings. The SE image (Fig. 1b) and the BSE image (Fig. 1c) were recorded using an electron beam energy of $15 \mathrm{keV}$ and a probe current of $5 \mathrm{nA}$ with a sample tilt of $70^{\circ}$ in a Merlin (Zeiss) FE-SEM. The same SEM is also used to obtain the experimental EBSD patterns shown in Figs $2 \mathrm{~b}, 3 \mathrm{a}$ and $3 \mathrm{~b}$ and EBSD maps displayed in Fig. 6. The BSE image shown in Figs $1 \mathrm{c}$ and $6 \mathrm{a}$ were taken combining the signals from three detectors, a multi-detector system (ARGUS TM) which was positioned below the phosphor screen to collect the forward scattered electrons. The Kikuchi bands ROI asymmetry image (Fig. 4a) and the IPF maps (Figs 4c, 5a and 5c) were obtained using a Bruker e-Flash ${ }^{\mathrm{HR}+}$ EBSD detector mounted on a LEO 1530VP (Zeiss) FE-SEM. The EBSD patterns were acquired using electron beam energy of $20 \mathrm{keV}$ and a probe current of $5 \mathrm{nA}$. The detector to sample distance was $18 \mathrm{~mm}$. The maps were obtained with a step size of $30 \mathrm{~nm}$ from EBSD patterns with a resolution of $160 \times 115$ pixels with $15 \mathrm{~ms}$ acquisition time. The mean angular error maps shown in Fig. $6 \mathrm{c}$ and $\mathrm{d}$ were also obtained using a Bruker e-Flash ${ }^{\mathrm{HR}+}$ EBSD detector in a Merlin (Zeiss) FE-SEM with electron beam energy of $20 \mathrm{keV}$ and a probe current of $5 \mathrm{nA}$ with a detector to sample distance of $21 \mathrm{~mm}$. The maps were acquired with a step size of $110 \mathrm{~nm}$ with a EBSD pattern resolution of $800 \times 576$ with $300 \mathrm{~ms}$ acquisition time. 
Cross-correlation coefficient ( $r$ ). For quantifying the agreement between two EBSD patterns, we have used the normalised cross-correlation coefficient $r$ which can be defined by the below formula.

$$
\mathrm{r}=\frac{\sum_{i, j}[f(i, j)-\bar{f}] \cdot[\omega(i, j)-\bar{\omega}]}{\sqrt{\sum_{i, j}[f(i, j)-\bar{f}]^{2}} \cdot \sqrt{\sum_{i, j}[\omega(i, j)-\bar{\omega}]^{2}}}
$$

where $f(i, j)$ and $\omega(i, j)$ are the pixel intensity values of the corresponding ROI in the two EBSD patterns to be compared whereas $\bar{f}$ and $\omega$ are the mean values in these ROIs. The absolute value of $r$ is in the range between 0 and 1 and does not depend on scale changes in the intensity of both patterns. Values of $r>0.6$ like those observed in this study indicate convincing fits between the experimental and simulated EBSD pattern $s^{38}$.

Assessment of APBs density. We have estimated the APBs density based on number of boundaries (intersection between green to blue region from the IFP maps) that crosses along APDs. We have taken 5 line scans from random locations and counted the numbers of boundaries crossing the $10 \mu \mathrm{m}$ in length and divided the number of boundaries by $10 \mu \mathrm{m}$, thus came up with an average APBs density of $\approx 2.6 \mu \mathrm{m}^{-1}$ from an scanned area of $\approx 75 \mu \mathrm{m}^{2}$.

Data availability. The data that support the findings of this study can be found online under DOI: http:// dx.doi.org/10.15129/2bb2bf6a-8ced-4c3f-812b-2b780f353b43 Alternatively, it is also available from the corresponding author on request.

\section{References}

1. Read Jr., W. T. Dislocations in crystals, New Jersey 1953.

2. Van Tendeloo, G., Lebedev, O. I. \& Amelinckx, S. Atomic and microstructure of CMR materials. Journal of Magnetism and Magnetic Materials 211, 73-83 (2000)

3. Yan, M., De Graef, M., Picard, Y. N. \& Salvador, P. A. Electron channeling contrast imaging of anti-phase boundaries in coherently strained $\mathrm{La}_{0.7} \mathrm{Sr}_{0.3} \mathrm{MnO}_{3}$ thin films on (110)-oriented $\mathrm{SrTiO}_{3}$. Applied Physics Letters 107, 041601-1-041601-5 (2015).

4. Beyer, A. et al. Atomic structure of (110) anti-phase boundaries in GaP on Si (001). Applied Physics Letters 103, 032107-1-032107-4 (2013).

5. Kemper, R. M. et al. Anti-phase domains in cubic GaN. Journal of Applied Physics 110, 123512-1-123512-5 (2011).

6. Khazaka, R. et al. Realization of minimum number of rotational domains in heteroepitaxied Si (110) on 3C SiC (001). Applied Physics Letters 108, 011608-1-011608-4 (2016).

7. Marcinkowski, M. J. \& Brown, N. Direct Observation of Antiphase Boundaries in the $\mathrm{Fe}_{3} \mathrm{Al}$ Superlattice. Journal of Applied Physics 33, 537-552 (1962).

8. Venkateswaran, S. P., Nuhfer, N. T. \& De Graef, M. Anti-phase boundaries and magnetic domain structures in Ni ${ }_{2} \mathrm{MnGa}^{-t y p e}$ Heusler alloys. Acta Materialia. 55, 2621-2636 (2007).

9. Singamaneni, S. R., Prater, J. T. \& Narayan, J. Multifunctional epitaxial systems on silicon substrates. Applied Physics Reviews 3, 031301-1-031301-38 (2016).

10. Look, J. R. \& Sizelove, D. C. Dislocation Scattering in GaN. Physical Review Letters 82, 1237 (1999).

11. R. A. Schwarzer, D. P. Field, B. L. Adams, M. Kumar, and A. J. Schwartz, in Electron Backscatter Diffraction in Materials Science, edited by A. J. Schwartz, M. Kumar, B. L. Adams, and D. P. Field (Springer, New York, 2009).

12. Wisniewski, W., Volksch, G. \& Russel, C. The degradation of EBSD-patterns as a tool to investigate surface crystallized glasses and to identify glassy surface layers. Ultramicroscopy 106, 307-313 (2006).

13. Chadi, D. J. Stabilities of Single-Layer and Bilayer Steps on Si (001) Surfaces. Physical Review Letters 59, 1691-1694 (1987).

14. Nemeth, I., Kunert, B., Stolz, W. \& Volz, K. Ways to quantitatively detect antiphase disorder in GaP films grown on Si(001) by transmission electron microscopy. Journal of Crystal Growth 310, 4763-4767 (2008).

15. Kunert, B., Nemeth, I., Reinhard, S., Volz, K. \& Stolz, W. Si (001) surface preparation for the antiphase domain free heteroepitaxial growth of GaP on Si substrate. Thin Solid Films 517, 140-143 (2008).

16. Beyer, A. et al. GaP heteroepitaxy on $\mathrm{Si}(001)$ : Correlation of $\mathrm{Si}$-surface structure, GaP growth conditions, and $\mathrm{Si}-\mathrm{III} / \mathrm{V}$ interface structure. Journal of Applied Physics 111, 083534-1-083534-6 (2012).

17. Beyer, A. et al. Influence of crystal polarity on crystal defects in GaP grown on exact Si (001). Journal of Applied Physics 109, 083529-1-083529-6 (2011).

18. Volz, K. et al. GaP-nucleation on exact Si (001) substrates for III/V device integration. Journal of Crystal Growth 315, 37-47 (2011).

19. Soga, T., Jimbo, T. \& Umeno, M. Growth and characterization of two-dimensional GaP on Si by metalorganic chemical vapor deposition. Journal of Crystal Growth 146, 554-557 (1995).

20. Ernst, F. \& Piroux, P. Formation of planar defects in the epitaxial growth of GaP on Si substrate by metalorganic chemical-vapor deposition. Journal of Applied Physics 64(9), 4526 (1988).

21. Le'toublon, A. et al. X-ray study of antiphase domains and their stability in MBE grown GaP on Si. Journal of Crystal Growth 323 , 409-412 (2011).

22. Doscher, H. \& Hannappel, T. In situ reflection anisotropy spectroscopy analysis of heteroepitaxial GaP films grown on Si(100). Journal of Applied Physics 107, 123532 (2010).

23. Doscher, H. et al. Indirect in situ characterization of $\mathrm{Si}(100)$ substrates at the initial stage of III-V heteroepitaxy. Journal of Crystal Growth 315, 16-21 (2011).

24. Pond, R. C. et al. Materials Research Society Proceedings 25, 273 (1983).

25. Kuan, T. S. \& Chang, C. A. Electron microscope studies of a Ge-GaAs superlattice grown by molecular beam epitaxy. Journal of Applied Physics 54, 4408-4413 (1983).

26. Goldstein, J., et al. Scanning Electron Microscopy and X-ray Microanalysis (Springer, New York, 2003).

27. Stefan Zaefferer, S. \& Elhami, N. Theory and application of electron channelling contrast imaging under controlled diffraction conditions. Acta Materiallia 75, 20-50 (2014).

28. Joy, D. C., Newbury, D. E. \& Davidson, D. L. Electron channeling patterns in the scanning electron microscope. Journal of Applied Physics 53, 81-122 (1982).

29. Holt, D. B. and Joy, D. C. SEM microcharacterization of semiconductors, Academic Press, London (1989).

30. Winkelmann, A., Trager-Cowan, C., Sweeney, F., Day, A. P. \& Parbrook, P. J. Many-beam dynamical simulation of electron backscatter diffraction patterns. Ultramicroscopy 107, 414-421 (2007).

31. Callahan, P. G. \& De Graef, M. Dynamical Electron Backscatter Diffraction Patterns. Part I: Pattern Simulations. Microscopy and Microanalysis 19, 1255-1265 (2013).

32. Winkelmann, A., Nolze, G., Vos, M., Salvat-Pujol, F. \& Werner, W. S. M. Physics-based simulation models for EBSD: advances and challenges. Materials Science and Engineering 109, 012018-1-012018-13 (2016). 
33. Baba- Kishi, K. Z. Electron backscatter Kikuchi diffraction in the scanning electron microscope for crystallographic analysis. Journal of Materials Science 37, 1715-1746 (2002).

34. Wilkinson, A. J. \& Britton, T. B. Strains, planes, and EBSD in materials science. Materials Today. 15, 366-376 (2012).

35. Nolze, G. \& Winkelmann, A. Exploring structural similarities between crystal phases using EBSD pattern comparison. Crystal Research Technology 49, 490-501 (2014).

36. Winkelmann, A. \& Nolze, G. Chirality determination of quartz crystals using Electron Backscatter Diffraction. Ultramicroscopy. 149, 58-63 (2015).

37. Engler, O. and Randle, V. Introduction to Texture Analysis: Macrotexture, Microtexture, and Orientation Mapping, 2nd ed. (CRC Press, Boston, MA, USA, 2009).

38. Gonzalez, R. C. and Woods, R. E. Digital Image Processing, 2nd ed. (Addison-Wesley Longman Publishing Co., Inc., Boston, MA, USA, 1992).

39. Winkelmann, A. \& Nolze, G. Point-group sensitive orientation mapping of non-centrosymmetric crystals. Applied Physics Letters 106, 072101-1-072101-4 (2015).

40. Winkelmann, A. Dynamical effects of anisotropic inelastic scattering in electron backscatter diffraction. Ultramicroscopy. 108, 1546-1550 (2008).

41. Vespucci, S. et al. Digital direct electron imaging of energy-filtered electron backscatter diffraction patterns. Physical Review B 92, 205301-1-205301-9 (2015).

42. Nolze, G., Grosse, C. \& Winkelmann, A. Kikuchi pattern analysis of noncentrosymmetric crystals. Journal of Applied Crystallography 48, 1405-1419 (2015).

43. Vos, M. \& Winkelmann, A. Element-resolved Kikuchi pattern measurements of non-centrosymmetric materials. Materials Characterization. 123, 328-338 (2017).

44. Wright, S., Nowell, M. M., Kloe, R., Camus, P. \& Rampton, T. Electron imaging with an EBSD detector. Ultramicroscopy. 148, $132-145$ (2015).

45. Payton, E. J. \& Nolze, G. The Backscatter Electron Signal as an Additional Tool for Phase Segmentation in Electron Backscatter Diffraction. Microscopy and Microanalysis 19, 929-941 (2013).

46. Schwarzer, R. A. \& Hjelen, J. Backscattered Electron Imaging with an EBSD Detector. Microscopy Today 23, 12-17 (2015).

47. Simkin, B. A. \& Crimp, M. A. An experimentally convenient configuration for electron channeling contrast imaging. Ultramicroscopy 77, 65-75 (1999).

48. Schwarzer, R. A. \& Sukkau, J. Electron Back Scattered Diffraction: Current State, Prospects and Comparison with X-Ray Diffraction Texture Measurement. The Banaras Metallurgist 18, 1-11 (2013).

49. Tao, X. \& Eades, A. Another way to implement diffraction contrast in SEM. Microscopy and Microanalysis 8, 690-691 (2002).

50. Winkelmann, A. et al. Diffraction effects and inelastic electron transport in angle-resolved microscopic imaging applications. Journal of Microscopy 00, 1-17 (2017).

51. Jussila, H. et al. Growth and characterization of GaP layers on silicon substrates by metal-organic vapour phase epitaxy. Physica Status Solidi C 9, 1607-1609 (2012).

52. Jussila, H., Nagarajan, S., Huhtio, T., Lipsanen, H. \& Tuomi, T. O. Structural study of GaP layers on misoriented silicon (001) substrates by transverse scan analysis. Journal of Applied Physics 111, 043518-1-043518-6 (2012).

53. Barrett, C. S. C. et al. Quantitative correlation of interfacial contamination and antiphase domain boundary density in GaAs on $\mathrm{Si}(100)$. Journal of Materials Science 51, 449-456 (2016).

54. Wilkinson, A. J., Meaden, G. \& Dingley, D. J. High-resolution elastic strain measurement from electron backscatter diffraction patterns: New levels of sensitivity. Ultramicroscopy 106, 307-313 (2006).

55. Wilkinson, A. J., Meaden, G. \& Dingley, D. J. High resolution mapping of strains and rotations using electron backscatter diffraction. Materials Science and Technology 22, 1271-1278 (2006).

56. Snedecor, G. W. and Cochran, W.G. "Statistical Methods", Wiley-Blackwell, 1989.

57. Vilalta-Clemente, A. et al. Cross-correlation based high resolution electron backscatter diffraction and electron channelling contrast imaging for strain mapping and dislocation distributions in InAlN thin films. Acta Materialia 125, 125-135 (2017)

\section{Acknowledgements}

G. Naresh-Kumar would like to thank Scottish University Physics Alliance (SUPA) for funding short term research visit to the Aalto University, Finland. This work was supported by the EPSRC project EP/M015181/1, "Manufacturing nanoGaN" and EP/P015719/1 "Quantitative non-destructive nanoscale characterisation of advanced materials".

\section{Author Contributions}

G.N.K., H.J. and A.W. designed the project; A.V.C. and G.N. performed EBSD experiments; H.J. and S.N. prepared samples, performed A.F.M. experiments and helped in writing the manuscript. A.W., A.V.C., G.N.K., analysed the data and G.N.K. wrote the paper. S.V. helped in analysing the data and discussed the results, A.J.W. and C.T.C. supervised the project. All the authors read and commented on the manuscript.

\section{Additional Information}

Competing Interests: The authors declare that they have no competing interests.

Publisher's note: Springer Nature remains neutral with regard to jurisdictional claims in published maps and institutional affiliations.

Open Access This article is licensed under a Creative Commons Attribution 4.0 International License, which permits use, sharing, adaptation, distribution and reproduction in any medium or format, as long as you give appropriate credit to the original author(s) and the source, provide a link to the Creative Commons license, and indicate if changes were made. The images or other third party material in this article are included in the article's Creative Commons license, unless indicated otherwise in a credit line to the material. If material is not included in the article's Creative Commons license and your intended use is not permitted by statutory regulation or exceeds the permitted use, you will need to obtain permission directly from the copyright holder. To view a copy of this license, visit http://creativecommons.org/licenses/by/4.0/.

(C) The Author(s) 2017 